\title{
Alles anders? - Welpenförderung während einer Pandemie
}

\author{
Celina del Amo
}

\author{
Homeoffice, lange Mittagspause, keine Abendtermine - für viele Menschen ideal, um den \\ langgehegten Wunsch nach einem Hund endlich umzusetzen. Doch wie lässt sich ein Welpe \\ auch unter Pandemiebedingungen zu einem unkomplizierten Begleiter ausbilden?
}

Seit dem ersten Corona-Lockdown im Frühjahr 2020 hat die Hundehaltung Hochkonjunktur. Auch die Übernahme von Welpen ist sprunghaft angestiegen. So günstig die Zeit für die Aufnahme eines Hundes als Familienmitglied auch erscheinen mag, so problembehaftet kann das Ganze sein. Angefangen bei der Auswahl eines Welpen bis hin zu den Möglichkeiten der Förderung ist zurzeit nämlich nichts mehr so wie es einmal war. Natürlich gibt es aber trotz aller Einschränkungen gute Ansatzpunkte, um den Start in das gemeinsame Leben in nachhaltiger Art erfolgreich verlaufen zu lassen.

\section{Über die Pandemie hinaus gedacht}

Auch wenn ungewiss ist, wie lange uns stärkere pandemiebedingte Einschränkungen begleiten werden, so ist es doch sehr wahrscheinlich, dass ein Hundeleben weit darüber hinaus reicht. Die Entscheidung, einen Hund bei sich aufzunehmen, will also wohl überlegt sein. Sie sollte gedanklich auf einen Zeitraum von 10 bis 15 Jahren bezogen werden.

Konkret geht es um folgende Fragen: Wird man langfristig genug Zeit für den Hund aufbringen können? Wie wird es mit dem Hund sein, wenn der Arbeitsschwerpunkt nicht mehr das Homeoffice ist? Was soll der Hund lernen bzw. was soll er als erwachsenes Tier leisten können? Sind diese Ziele (beispielsweise ein entspannter und freundlicher Umgang mit fremden Menschen oder ein problemloses Alleinebleiben für 2-3 Stunden) unter den Pandemiebedingungen überhaupt zu erreichen? Welche Besonderheiten gibt es hierbei eventuell zu berücksichtigen?

\section{Angebot und Nachfrage}

Zurzeit haben wir es mit folgender Situation zu tun: Eine größere Anzahl erfahrener Züchter hat geplante Würfe verschoben oder die Kontaktaufnahme durch Neuinteressenten eingeschränkt, um der teils undurchdacht erscheinenden Hundewunsch-Flut zu entgehen. Gleichzei- tig hat die hohe Nachfrage nach Welpen aber jede Menge schwarze Schafe auf den Plan gerufen. Je spontaner man an einen Welpen kommt, desto höher liegt aktuell die Chance, dass es sich um einen Hund aus einer wahllosen Verpaarung von leider oftmals gesundheitlich und/oder psychisch ungeeigneten Elterntieren handelt. Vor allem Hundeneulinge starten so oftmals unter schwierigen Bedingungen in das Leben mit einem Hund. In der Mehrzahl der Fälle auch ohne sich dieser Besonderheit und Problematik bewusst zu sein.

\begin{abstract}
Merke
Die zurzeit vielleicht günstig erscheinenden Grundbedingungen (speziell das Homeoffice) sollten niemanden bei der Auswahl eines Hundes zur Eile antreiben.
\end{abstract}

Auf einen wirklich passenden Hund auch einmal zu warten, zahlt sich später oftmals doppelt und dreifach aus. Scheinbare „Schnäppchen“ oder Spontankäufe erweisen sich hingegen leider häufig als zukünftige Sorgenkinder.

\section{Förderung im neuen Zuhause}

Ganz grundsätzlich gilt es, sich ab dem Tag des Einzugs eines Welpen auch den Erziehungsfragen zu widmen. Die Bedingungen, die sich dem neuen Tierhalter bieten, sind überaus günstig. Welpen sind nämlich wahre „Lernmonster“. Sie saugen bereitwillig alle Informationen in sich auf. Überzeugungskraft ist also weniger gefordert. Vielmehr besteht die Kunst der Hundeerziehung im Grunde darin, die Erfahrungen, die der Welpe macht, so zu steuern, dass er sich zu genau dem Hund entwickeln kann, den man sich erhofft hat.

Ob der Hund viele oder nur wenige Kommandos lernt, ist hierbei tatsächlich zweitrangig. Im Vordergrund steht, wie er auf emotionaler Ebene seine Umwelt wahrnimmt und welche Verhaltensreaktionen durch die erlebten Emotionen angetriggert werden. Entscheidend ist das den Trainingsbemühungen zugrunde liegende Konzept. 


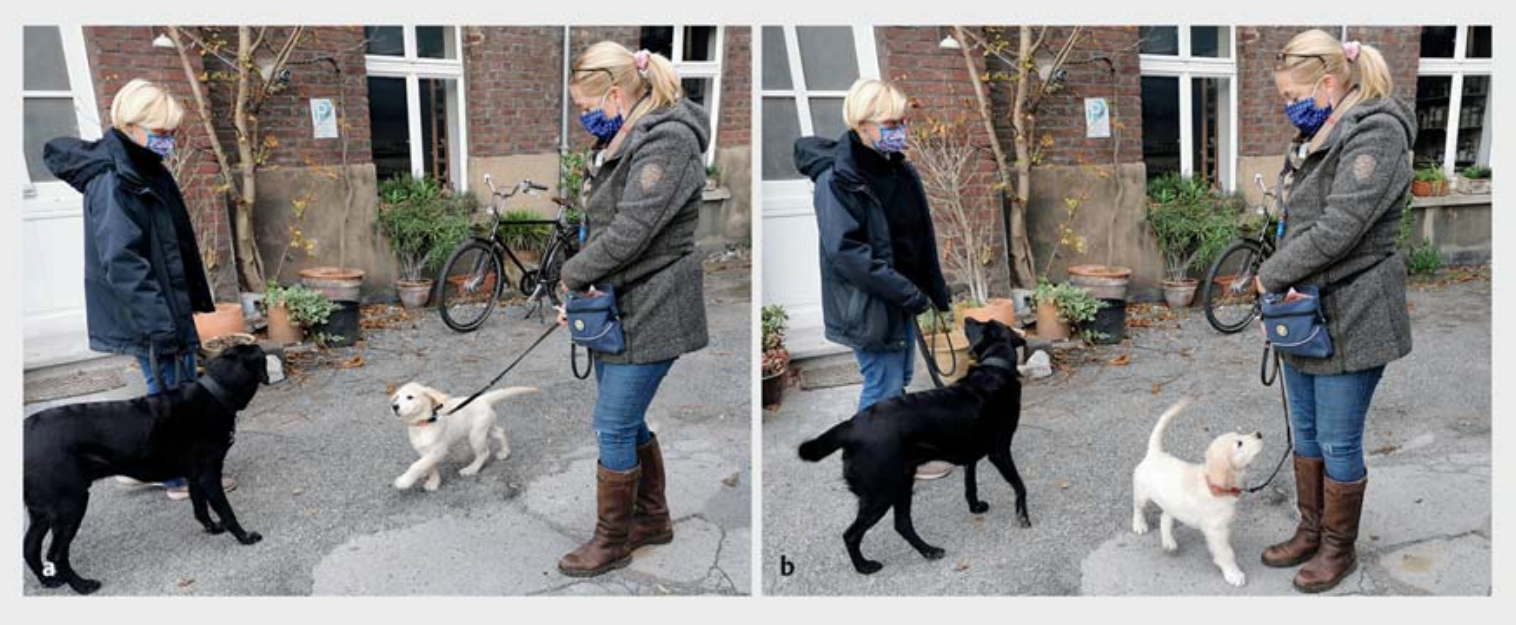

- Abb. 1 Hundekontakte: Gezielte Verabredungen mit einem freundlichen, souveränen und wohlerzogenen Artgenossen helfen dem Welpen in seiner emotionalen Entwicklung. Quelle: Celina del Amo

Dieses sollte gut durchdacht sowie kleinschrittig aufgebaut sein und belohnungsbasiert umgesetzt werden.

Dinge wie die allgemeine Kooperationsbereitschaft des Hundes, der Aufbau von Konzentration, Ruhe und Geduld sowie der Rückruf und ein freudiges und promptes Auslassen von Gegenständen sollten weit oben auf der Trainingsliste stehen, da ein gutes Gelingen dieser Details einem problemfreien Zusammenleben ausgesprochen zuträglich ist.

Modern umgesetzte trainerische Einflussnahme hat nichts mit Drill zu tun. Sie kann vielmehr spielerisch und in den Alltag integriert erfolgen.

Merke

Beim Training zählen ein durchdachtes Vorgehen und die nötige Konsequenz bei der Umsetzung.

\section{Sozialisation mit Menschen und Artgenossen}

Die pandemiebedingten Einschränkungen stellen in Bezug auf die Förderungsmöglichkeiten von Welpen aktuell eine Besonderheit dar. So sind vor allem Kontakte mit fremden bzw. nicht zum Haushalt gehörenden Personen weniger leicht verfügbar. Dies muss jedoch nicht grundsätzlich als Nachteil betrachtet werden. Verleitungen und auch regelrechte Belästigungen, die oftmals in zufälligen Kontakten eine Rolle spielen, fallen nicht mehr so stark ins Gewicht. Es ist daher zurzeit sogar leichter, mit dem Welpen ein gesittetes An-Menschen-Vorbeigehen zu üben und in der Nähe von Menschen (jedoch in dem Fall ohne persönlichen Kontakt) Ruhe-Übungen umzusetzen. Auch für Hundekontakte gilt, dass eine gute Sozialisation durchaus möglich ist. Wen der Welpe wann kennenlernt, kann der Tierhalter individuell steuern.

\section{Direkte Kontakte mit Menschen}

Für direkte persönliche Kontakte muss und sollte allerdings etwas mehr Planungsarbeit aufgebracht werden. Hier ist das Ziel, dass der Welpe Menschen beiderlei Geschlechts und verschiedener Altersstufen kennenlernen kann. Durch zielgerichtete Verabredungen können diese Kontakte ermöglicht werden. Ideal ist es, bei diesen Schulungsübungen auf Personen zurückzugreifen, die die eigene Erziehungsarbeit nicht torpedieren, sondern sich dem Welpen gegenüber schlicht neutral und freundlich verhalten. So fällt es auch hier leicht, dem Welpen zu vermitteln, dass persönliche Kontakte (drauBen und nach Möglichkeit auch im Sinne einer Besuchssituation zuhause) ebenfalls an Regeln gebunden sind und er mit ruhigem Verhalten den meisten Erfolg einheimsen kann (d. h. er darf Kontakt aufnehmen und erhält ein positives, aber „actionarmes“ Feedback von der Kontaktperson oder Futterbelohnungen für ruhiges oder braves Verhalten vom Tierhalter).

\section{Hundekontakte}

Im Sinne der sozialen Schulung gilt ganz Ähnliches für Hundekontakte. Denn auch hier zählt im Hinblick auf die Entwicklung des Welpen nicht allein die Anzahl an Hundekontakten, sondern vielmehr deren Qualität bzw. das emotionale Erleben des Welpen. Bekanntschaften mit guten Lehrmeistern (freundliche, souveräne und wohlerzogene Hunde) sind Gold wert. Damit diese häufig genug stattfinden können, sind gezielte Verabredungen zurzeit die beste Wahl. Natürlich können diese - sofern es bei der Umsetzung keine Beschränkungen (mehr) gibt - auch in einer mit Bedacht organisierten und fachkundig angeleiteten Trainingsgruppe stattfinden. Das Lernziel ist, den Welpen in ruhigem und höflichem Hundeverhalten zu schulen. 
Als ungeeignet sind Kontakte zu bezeichnen, bei denen der Welpe sich fürchtet oder sich Artgenossen gegenüber aufdringlich, wüst, frech oder gar aggressiv verhält. Sollte dies zu beobachten sein, tut der Tierhalter gut daran, dies ohne Strafeinsatz umgehend in andere Bahnen zu lenken.

Eine wichtige positive steuernde Einflussnahme bezieht sich darauf, den Welpen darin zu unterstützen, sich in Anwesenheit von Artgenossen gesittet zu verhalten und sich immer wieder auch von ihnen ab- und dem Halter zuzuwenden ( $\bullet$ Abb. 1). Gleichzeitig sollte eine explizite Auswahl getroffen werden, mit welchen Hunden der Welpe wie lange und in welcher Art Kontakt haben darf. Wie bei allen Details in der Verhaltensentwicklung gilt schließlich auch hier: Übung macht den Meister - im Guten wie im Schlechten!

\section{Merke}

Die gezielte Verabredung und Steuerung von sozialen Kontakten ist ein wichtiges Detail, um den Hund im Heranwachsen „auf dem richtigen Weg“ zu halten.

\section{Besonderheiten der Welpenförderung während der Corona-Pandemie}

Die meisten Übungen und Welpenförderungsmaßnahmen können auch während bestehender Einschränkungen (ggf. mit leichten Abwandlungen) umgesetzt werden. In den folgenden Bereichen kommt es aber zu pandemiebedingten Besonderheiten. Für eine langfristig problemfreie gemeinsame Zeit lohnt es sich, diese Dinge von Anfang an zu berücksichtigen.

\section{Alleine bleiben will gelernt sein}

Welpen fühlen sich sicher und geborgen, wenn dort, wo sie sich aufhalten, Familienmitglieder anwesend sind, an die sie sich bereits gebunden haben. In engem Verband mit vertrauten Individuen zu leben, ist ihnen nämlich nicht nur seit jeher bekannt, sondern es entspricht grundsätzlich dem „Rudeltiernaturell“. Stehen die vertrauten Artgenossen nach dem Umzug ins neue Zuhause nicht (mehr) zur Verfügung, verlagert sich ihr Geborgenheitsbedürfnis in Richtung des neuen Tierhalters. In der Hundehaltung ergibt sich langfristig irgendwann die Notwendigkeit, den Hund auch einmal für eine Zeit lang alleine zu lassen. Das Alleinsein sollte daher gut geübt werden.

Tierhalter, die im Homeoffice tätig sind, verbringen aktuell viel gemeinsame Zeit mit ihrem Hund. Für den Welpen ist dies zunächst natürlich schön und auch für den Tierhalter ist kein Problem ersichtlich. Von allergrößter Wichtigkeit ist jedoch im Sinne einer bestmöglichen Problemprophylaxe, den Welpen bereits jetzt täglich in kleinschrittiger Art an das Alleinesein zu gewöhnen. Diese Übungen können auch umgesetzt werden, wenn gar kei-

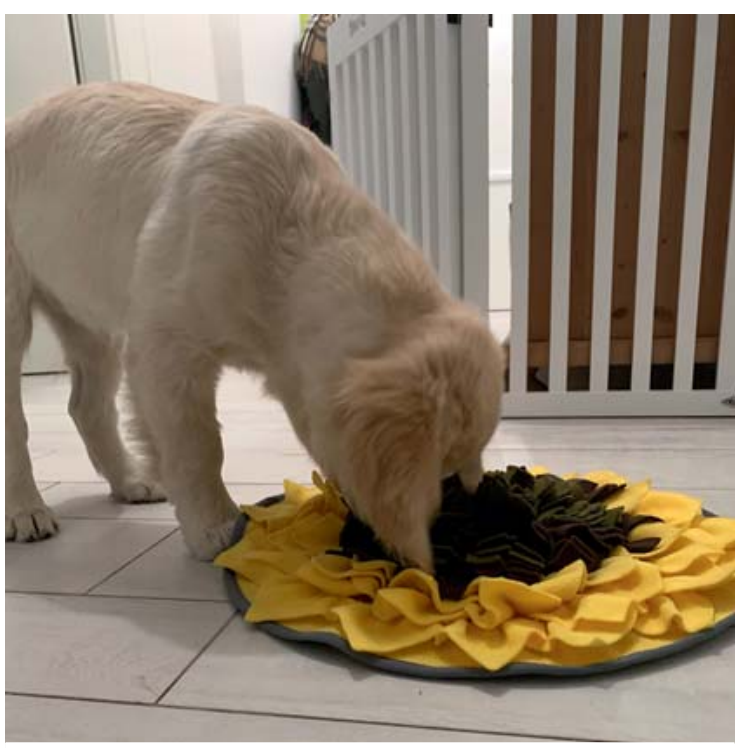

- Abb. 2 Distanztraining mit einem Schnüffelteppich macht Spaß. Quelle: Celina del Amo

ne Notwendigkeit besteht, das Haus zu verlassen oder wenn sogar offizielle Ausgeh-Restriktionen einzuhalten sind.

Die auf Welpenlevel umgesetzten Trennungsübungen bewegen sich anfangs im unteren Minutenbereich. Es geht vorrangig um den Erhalt von Angstfreiheit bei räumlicher Trennung von wichtigen Sozialpartnern, nicht bereits um reale Isolationen. Erst nach und nach ist es sinnvoll, die Zeiten auf ein im Alltag realistisches Maß zu verlängern und „echte“ Trennungsübungen in Angriff zu nehmen ( $\triangleright$ Abb. 2).

\section{WICHTIG!}

Regelmäßig stundenlange Isolationsphasen sind im Sinne einer tierschutzrechtlich einwandfreien Unterbringung für ein obligat sozial ausgerichtetes Tier wie den Hund stets kritisch zu betrachten. Beim Thema „Alleinsein“ geht es nicht nur um die coronabedingten Besonderheiten, sondern auch um eine langfristig hundekonforme Planung.

\section{Das Aufnehmen von Unrat}

Viele Welpen tendieren dazu, alle möglichen herumliegenden Dinge aufzunehmen. Bezieht sich dieses Verhalten auch auf Taschentücher oder Gebrauchsmasken, die der Welpe draußen findet, entsteht vielen Tierhaltern zurzeit besonderer Stress. Schnell kommt es dann zu Konfliktsituationen. Grundsätzlich bezieht sich das angesprochene Training aber auf Dinge aller Art, also auch auf herumliegende Essensreste, Zigarettenstummel, Glasstücke etc. 


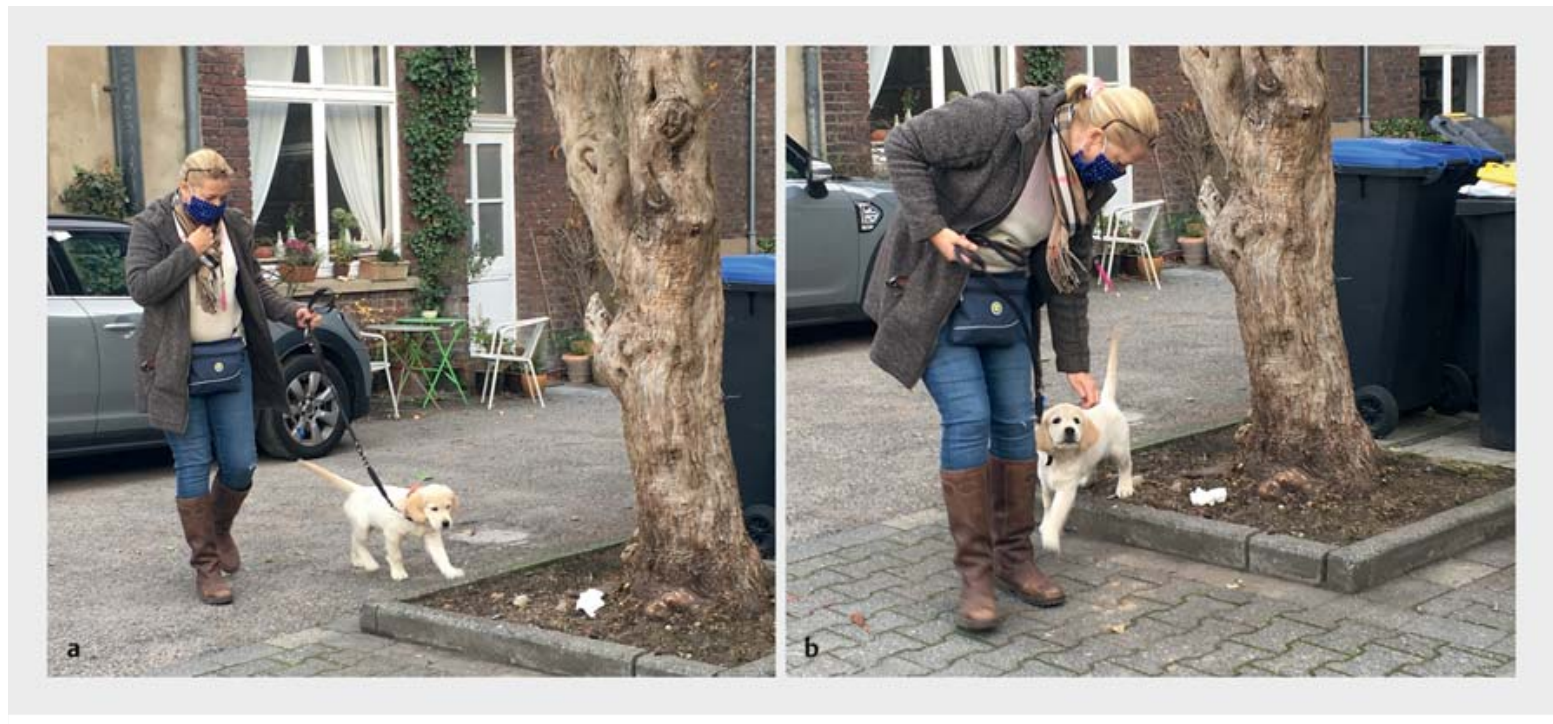

- Abb. 3 Aufnehmen von Unrat: Ein schnelles Ablenken des Welpen durch die direkte Ansprache hilft, damit der Welpe das auf dem Boden liegende Taschentuch unwichtig findet. Auf diese Weise kann die Hundehalterin diese Verleitungssituation unkompliziert auflösen. Quelle: Celina del Amo

Von Anfang an sollte mit dem Welpen (drinnen und draußen) geübt werden, an allen möglichen kleineren und potenziellen „Beutestücken“ vorbeigeführt zu werden, ohne ihnen Beachtung zu schenken. Im Aufbau der Übung und ganz allgemein in den ersten Wochen zahlt sich bezüglich etwaiger Verleitungen Weitblick aus. Es geht zunächst darum, den Welpen gar nicht erst in Verleitungssituationen zu führen, die man nur schwer kontrollieren kann! Hierbei spielt vor allem die Nähe zu der Verleitung eine wichtige Rolle. Zudem spricht nichts dagegen, den Welpen in möglichst unauffälliger Art (über Ansprache, Spiel, eine Übung) von interessant erscheinendem Unrat abzulenken ( $\boldsymbol{A}$ Abb. 3). Es ist sinnvoll, sämtliche Übungen so umzusetzen, dass der Welpe manipulations- und fehlerfrei die richtige Lösung finden kann.

Gleichzeitig sollte zielgerichtet ein freudiges AUS-Lassen trainiert werden. Schnelle und nachhaltige Erfolge werden erzielt, wenn das Signal AUS als Einleitung für ein aus Hundesicht hochwertiges Tauschgeschäft oder für den umgehenden Erhalt eines attraktiven Snacks eingeführt wird.

\section{Merke}

Ein streitfreies und freudig umgesetztes AUS lernt der Hund, wenn man ihm in den Übungen das Gefühl vermittelt etwas zu gewinnen, statt etwas zu verlieren.

\section{Welpenförderung in der Behandlungssituation}

Nicht nur im Privaten, sondern auch in der Praxissituation gibt es coronabedingte Besonderheiten für Welpen und deren Besitzer. Das Handling der Tiere in der Praxissituation liegt aktuell oftmals alleinig in den Händen des Praxisteams.
Für Welpen ist das eine große Herausforderung: Sie werden mit Dingen konfrontiert, die ihnen noch nicht vertraut und häufig zunächst einmal auch unheimlich (ggf. auch unangenehm) sind. Und das Ganze findet auch noch durch fremde Personen statt. Die Gefahr, dass sich hinsichtlich der Tierarztsituation Ängste manifestieren, ist größer denn je! Es gibt aber einen einfachen Ausweg aus diesem Dilemma, und zwar in der Form eines kleinschrittig und belohnungsintensiv umgesetzten Gedulds- und Kooperationstrainings. Dies geht gleichermaßen an die Adressen des Tierhalters als auch an die des Praxisteams.

Das Übungsprogramm sollte sich mindestens darauf beziehen, den Hund überall am Körper anfassen zu können, ohne Angst oder Abwehr auszulösen. Weiter gefasst lohnen sich sämtliche Übungen, die unter dem Begriff Medical Training zusammengefasst werden. Dieser Trainingsbereich erfreut sich schon seit einiger Zeit eines immer größeren Bekanntheitsgrads. Anleitungen für beide Parteien (Tierbesitzer und das Praxisteam) finden sich sowohl in Büchern als auch in Online-Kursen ( $\bullet$ Abb. 4).

\footnotetext{
Merke

Welpen mit allen Griffen und Maßnahmen vertraut zu machen, die für eine bestmögliche Pflege und Versorgung erforderlich sind, zahlt sich lebenslang aus.
}

\section{Fazit}

Die Covid-19-Pandemie hat direkte Auswirkungen auf das Zusammenleben mit Hunden. Bei der Förderung von Welpen kann und sollte darauf geachtet werden, ihnen in einigen Bereichen ganz spezielle Unterstützung zukommen zu lassen, um sie allumfassend auf ihr weiteres Le- 


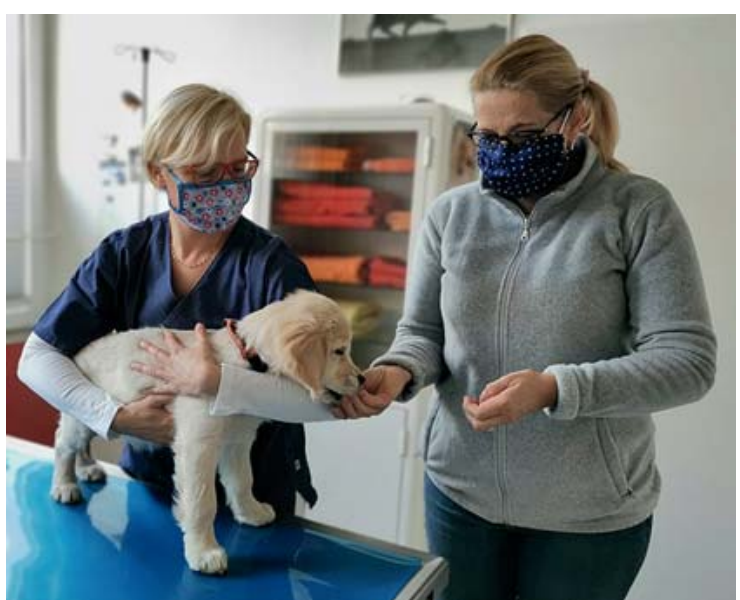

- Abb. 4 Auch unter Pandemiebedingungen können Welpen in kleinschrittigen, belohnungsintensiven Kooperationstrainings an den Besuch beim Tierarzt gewöhnt werden. Quelle: Celina del Amo

ben vorzubereiten. Die Maßnahmen, die hierfür erforderlich sind, sind bei entsprechender Planung allesamt mit einer minimalen Zeitinvestition umsetzbar. Sie basieren auf einer vorausschauenden Vorgehensweise und einem positiven Übungsaufbau.

\section{EXTRA}

Für interessierte Kollegen und Hundehalter gibt es den Online-Videokurs „Startrampe für Welpen“ unter Verwendung des Gutscheincodes „Pandemie“ bis zum 31.12.2021 zu einem Corona-Rabattpreis von 49,- EUR.

www.startrampe-fuer-welpen.de

\section{Korrespondenzadresse}

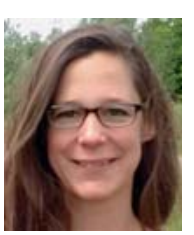

\section{Celina del Amo}

Tierärztliche Gemeinschaftspraxis Lupologic Fesserstraße 21

41462 Neuss

Deutschland

\section{Bibliografie}

Kleintier konkret 2020; 23: 19-23

DOI 10.1055/a-1308-5056

ISSN 1434-9132

(c) 2020. Thieme. All rights reserved.

Georg Thieme Verlag KG, Rüdigerstraße 14,

70469 Stuttgart, Germany 